\title{
Performance Evaluation Using Laser Doppler Vibrometer Sensing Technique on Advanced Lead Magnesium Niobate-lead Titanate Piezoelectric-material-based Microactuator for Hard Drive Head
}

\author{
Parinya Wattananukulchai and Don Isarakorn* \\ Department of Instrumentation and Control Engineering, Faculty of Engineering, \\ King Mongkut's Institute of Technology Ladkrabang, Bangkok 10520, Thailand \\ (Received December 15, 2017; accepted February 13, 2018)
}

Keywords: PZT, PMN-PT, HDD, HGA, TPI, resonance, stroke, DSA

This paper presents a microactuator for a dual-stage actuator (DSA) of hard disk drive (HDD) based on a lead magnesium niobate-lead titanate (PMN-PT) piezoelectric material and a comparative evaluation of its performance versus that of a traditional lead zirconate titanate (PZT) microactuator using the laser Doppler vibrometer (LDV) sensing technique. PZT microactuator technology has commonly been implemented in the read/write (R/W) magnetic head of HDD. It has a significant function, that is, it moves the magnetic head rapidly and accurately. In order to achieve both accurate positioning control on data tracks and high-speed access across another data track, advanced high-performance actuators and servo control technologies are necessary. An actuator with a wide stroke travel range is essential for HDD as it gives a high-speed access performance. In this study, we focused on comparing the proposed dual-stage PMN-PT head-based actuator with the existing PZT actuator that were mounted on a commercial head gimbal assembly (HGA) using an LDV to determine whether the proposed device could be a worthy replacement of the traditional PZT microactuator. Our experimental results show that the proposed PMN-PT microactuator provided more than twofold improvement in the stroke travel of the R/W magnetic head. Moreover, its dynamic behavior was suitable for assembling a high-density HDD in the future for big data storage without any needs to redesign the HGA nor to costly invest in new assembling machines in a production line. The comparative data of dynamic and static behaviors of PMN-PT versus PZT obtained in this study may be put to good use by sensor designers. Moreover, for many cyber-physical system designers, our data may make them take interest in PMN-PT as a better-performing sensor and an actuator for their systems.

\section{Introduction}

Setting a technological roadmap for the hard disk drive (HDD) industry is the key to expanding storage capacity for supporting the analysis of a huge volume of cyber data in cloud computing. One of the most challenging issues that is a consequence of tremendously increased 
areal density is that it requires extremely smaller HDD track widths. HDD track widths have been getting smaller at a rate of approximately $15 \%$ annually for concentric recording, ${ }^{(1)}$ in which bits are written in concentric segments across the radius of each disk. The write-read head in HDD is a mechanically positioned magnetic head. A radially traversing head reads data that are stored on concentric data tracks on a rotating disk. The recording density and spindle rotation speed of HDD have been developed and have advanced at a remarkable rate over the past decade, but the number of tracks per inch (TPI) has been much more difficult to develop. Fortunately, the number of bits per inch (BPI) can be increased by using highly advanced head and media technologies. ${ }^{(2)}$ An extremely precise track-following system is necessary for attaining higher track densities. A second-stage actuator for finely moving a read/write (R/W) magnetic head is considered to be a good solution that meets the higher accuracy and speed requirements of the more advanced servomechanism in today's HDD. ${ }^{(3,4)}$ For second-stage actuation, a precise actuator assembly is integrated into the head gimbal assembly (HGA) at a location near the magnetic heads and isolated from the voice coil motor (VCM) that is used to drive the whole actuator. To perform track-seeking, a conventional VCM moves the entire head and arm assembly at a low frequency, and the motion of the slider enables the microactuator mounted between the VCM and the slider head to perform high-frequency track-following. To meet this objective, various microactuators including piezoelectric, ${ }^{(5,6)}$ electromagnetic, ${ }^{(7)}$ and electrostatic ${ }^{(8,9)}$ ones have been developed.

In most solutions for second-stage actuators, the common piezoelectric material used is lead zirconate titanate $\left[\mathrm{Pb}(\mathrm{Zr}, \mathrm{Ti}) \mathrm{O}_{3}\right.$ or polycrystalline lead zirconate titanate (PZT)]. PZT actuators have a very long developmental history in terms of both material and design, but now their mechanical and electrical properties may not be suitable for the future development of today's HDD head: this is because a single-layer PZT plate cannot produce a large displacement. Moreover, although a multilayer PZT plate can provide a large displacement, its cost and manufacturing complexity are prohibitive. Recently, a new piezoelectric material, lead magnesium niobate-lead titanate (PMN-PT), which has good potential for obtaining a larger displacement, has been introduced. PMN-PT has been shown to provide a much better energy harvesting performance than PZT in a paper by Panthongsy and Isarakorn; ${ }^{(10)}$ it provided an energy density of $352.85 \mathrm{~J} / \mathrm{gm}^{3}$, while PZT provided only $8.44 \mathrm{~J} / \mathrm{gm}^{3}$. This is the main reason why we were interested in developing PMN-PT as an actuator because their result implies that, for a given applied voltage, PMN-PT should convert the electrical energy into a longer slider head travel. Even though PMN-PT materials have a high dielectric permittivity, high piezoelectric properties, and a high electrostriction, which are much higher than those of existing PZT materials, they have a low Curie temperature and thus were not widely used in HDD. However, a new supplier-developed PMN-PT material is currently available. Therefore, in this study, we aimed to comparatively evaluate the performance characteristics of PZT versus PMN-PT extensively as used in a real actuator. It was expected that PMNPT would provide a reasonable compromise between the static and dynamic behaviors of the actuator. In addition, the bandwidth of a microactuator crucially depends on the resonance mode: it determines the overall stability of the servo control system and the settling and seek performance characteristics of the HDD system. ${ }^{(11)}$ Therefore, in this study, the resonance 
mode of the proposed actuator was also determined experimentally whether it remained the same or changed to a certain degree. The comparative data of the dynamic and static behaviors of PMN-PT versus PZT obtained in this study may be put to good use by sensor designers. ${ }^{(12-14)}$

\section{Principles of HGA and Laser Doppler Vibrometer (LDV) Sensing Technique}

In 1976, the first commercially available HGA used a single-stage actuator with a VCM actuator to position the R/W head at the desired location and to reduce the seek time, as shown in Fig. 1(a). To increase the HDD data density, the size of the bits that represents a piece of information stored on the disk and the spacing between HDD concentric tracks were reduced. With these reductions, it became more difficult to position the R/W head's transducer element over the center of the data track. In addition, the actuator's arm and suspension that extend from the VCM to the R/W head swing the head from the load/unload ramp beyond the disk's outer diameter to the inner-diameter data track near the central motor hub. The relatively large mass of the head stack assembly results in a relatively low vibrational resonance frequency, which makes an accurate head positioning over high-density tracks very difficult and slow. For the next-generation HDD that can meet today's areal density target of much smaller track widths, a dual-stage actuator (DSA) is needed, as shown in Fig. 1(b). A new piezoelectric material in the DSA is required to meet the technology roadmap of higher areal density. ${ }^{(15)}$ The key to achieving a DSA design is choosing the right piezoelectric materials. Generally, polycrystalline PZT is used in manufacturing HDD as it is cheaper than single-crystalline PMN-PT. However, a new fabrication technology for a PMN-PT material that is as cheap as a PZT multilayer is now available. The physical and piezoelectric properties of both are shown in Table 1.

\subsection{Second-stage actuator in HGA}

A DSA implemented in a HGA consists of a conventional VCM first stage and a piezoelectric actuator second stage. The second stage consists of two piezoelectric actuators attached to a

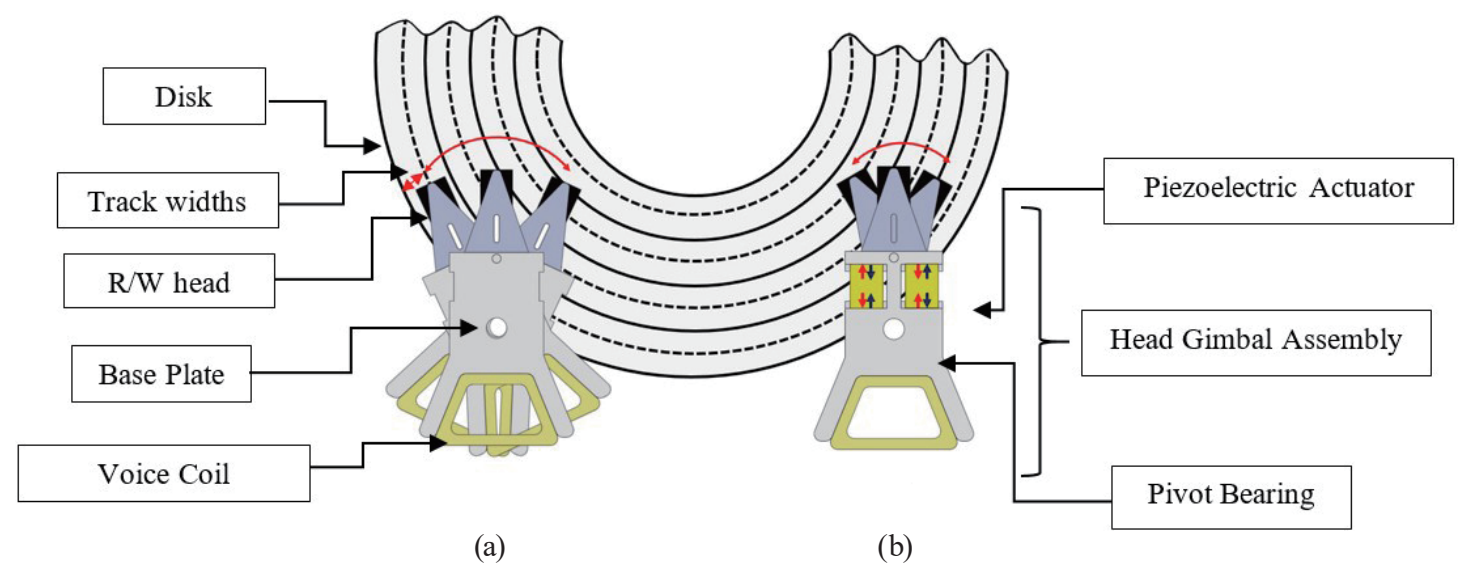

Fig. 1. (Color online) HGA designs: (a) single-stage actuator and (b) DSA. 
Table 1

Physical and piezoelectric properties.

\begin{tabular}{lccc}
\hline Composition & Unit $(\mathrm{N} / \mathrm{A})$ & PZT & PMN-PT \\
\hline Density $(p)$ & $\mathrm{kg} / \mathrm{m}^{2}$ & 7500 & 8200 \\
Dissipation $\left(D / \tan ^{\delta}\right)$ & $\mathrm{N} / \mathrm{A}$ & 0.025 & 0.01 \\
Elastic compliance $\left(S_{11}\right)$ & $\mathrm{pm}^{2} / \mathrm{N}$ & 10 & 14 \\
Electromechanical coupling coefficient $\left(k_{p}\right)$ & $\mathrm{N} / \mathrm{A}$ & 0.72 & 0.85 \\
Initial depolarization field $\left(E_{c}\right)$ & $\mathrm{V} / \mathrm{mil}$ & 20 & 38 \\
Mechanical Q $\left(Q_{m}\right)$ & $\mathrm{N} / \mathrm{A}$ & 30 & 42 \\
Piezoelectric coefficient $\left(d_{31}\right)$ & $\mathrm{pC} / \mathrm{N}$ & 250 & 620 \\
Polarization field $\left(E_{p}\right)$ & $\mathrm{V} / \mathrm{mil}$ & 65 & 70 \\
Relative dielectric constant $\left(K_{33}\right)$ & $\mathrm{N} / \mathrm{A}$ & 3200 & 4100 \\
\hline
\end{tabular}

suspension's baseplate in a robust design as shown in Fig. 1(a). The first-stage (coarse) actuator is characterized by a large travel range, but generally poor accuracy and slow response. The second-stage (fine) actuator moves more accurately with faster response but only for a limited travel range. By combining both actuators with an appropriate control strategy, the limitations of the single-stage actuator can be overcome; a combined performance of both high precision and large travel range can be achieved.

The operation of the second-stage actuator with voltage applied to the microactuator circuit is shown in Fig. 2(b); one piezoelectric die expands as the other contracts as shown in Fig. 2(a). An application of opposite voltages causes the slider to move in opposite directions. This action causes a $\mathrm{R} / \mathrm{W}$ head movement that is less than one millionth of a meter, but it is exquisitely finely controlled. Since the piezoelectric actuator stroke at the head element is very short and the moving mass is very small and light, this element's vibrational resonance frequency is much higher than that of the VCM single-stage actuator. As a result, the DSA can rapidly and accurately position the head element over the correct data track.

\subsection{Piezoelectric material selection}

For the past few years, the dual-stage positioning of a magnetic head in HDD by using a PZT microactuator has been favored. A piezoelectric PZT material can only actuate a very short displacement despite its advantages such as a high Curie temperature and long-term reliability. To move an R/W head through a large amount of data stored in HDD, an actuator that can cause a wide range of displacement is required. A single crystal of PMN-PT solid solution, $(1-x)\left[\mathrm{Pb}\left(\mathrm{Mg}_{1 / 3} \mathrm{Nb}_{2 / 3}\right) \mathrm{O}_{3}\right]-x\left[\mathrm{PbTiO}_{3}\right]$ (PMN-PT), is a new-generation piezoelectric material. This new material was formulated to exhibit a large electromechanical coupling coefficient, a large piezoelectric coefficient, a large dielectric constant, and a low dielectric loss. All of these properties result in the vast improvement of stroke bandwidth.

The single-crystal PMN-PT material exhibits desirable piezoelectric properties. If all of its domains were perfectly aligned, it can provide ten times the strain of a comparable polycrystalline PZT element. ${ }^{(16)}$ Nevertheless, its main weak points are its low Curie temperature and high cost. 

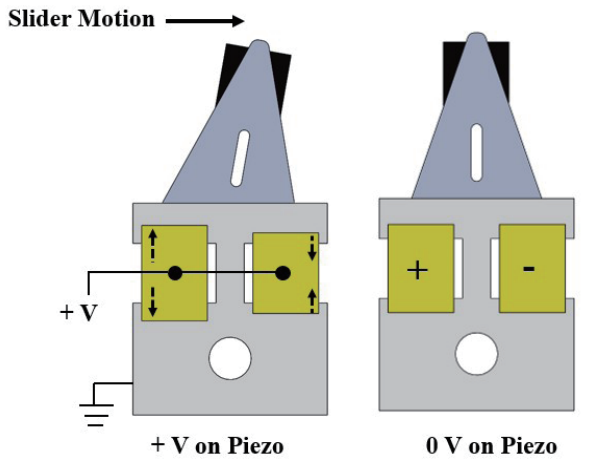

(a)
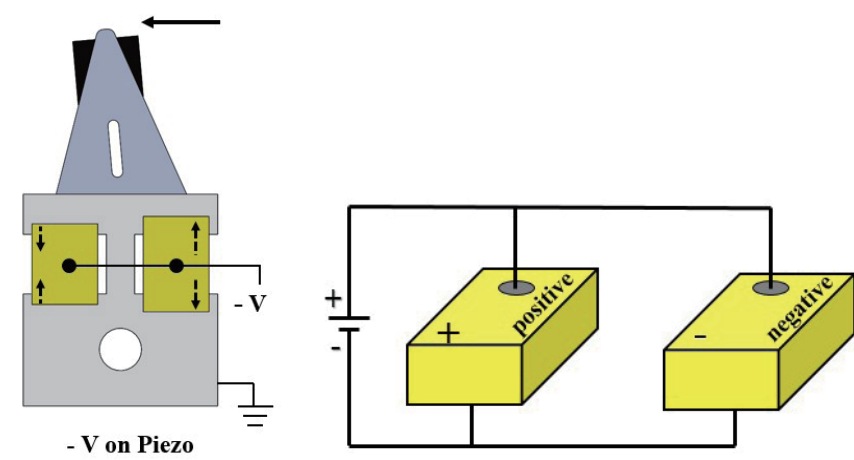

(b)

Fig. 2. (Color online) (a) R/W head movement of the second-stage piezoactuator in operation and (b) piezoelectric circuit.

Therefore, with a recent supplier development of a high-Curie-temperature and lowcost PMN-PT single crystal, it was found that PMN-PT was able to truly provide a larger displacement than PZT. This study is an extension of a previous simulation study of the displacement and resonance modes of $\mathrm{HGA},{ }^{(17)}$ which also suggest that the improvement could be expected. The dimensions of each layer of both piezoelectric materials are shown in Table 2. The cross-sectional electrode surface is plated with Ni chrome and Au electrodes [Figs. 3(a) and $3(b)]$.

Piezoelectric wafers were supplied and attached to UV release tape. It was easy to verify the poling direction; a label with either a positive $(+)$ or negative $(-)$ symbol was affixed to the tape to designate the positive or negative pole, respectively, as shown in Fig. 4(a), and a raw HGA nonattached piezoelectric is shown in Fig. 4(b).

\subsection{LDV sensing technique}

An LDV is a device for making noncontact measurements of a vibrating surface. A laser beam from an LDV is pointed at a surface of interest, and the reflected beam and its Doppler shift are extracted from the surface vibration amplitude and frequency that are the outcomes of the motion of the surface. The output of an LDV is a continuous analog voltage that is directly proportional to the target surface velocity component along the direction of the laser beam.

An LDV can be used in many types of measurement techniques that depend on a specific application purpose, such as single-point vibrometry, scanning vibrometry, three-dimensional (3D) vibrometry, rotational vibrometry, differential vibrometry, multibeam vibrometry, selfmixing vibrometry, and continuous scan laser Doppler vibrometry. In this study, we used a single-point vibrometer. It is a standard equipment for measuring the velocity of any point of a target surface under observation. In general, a laser interferometer uses two beams to measure the vibration of a target surface (a slider head in this study) with a frequency domain difference between the internal reference beam (laser signal input) and the test beam (laser signal output), as shown in Fig. 5(a). The raw data from the LDV is shown in Fig. 5(b), where the $x$-axis is the data point and the $y$-axis is the position of the moving slider head. 
Table 2

Piezoelectric material dimensions and tolerance specifications.

\begin{tabular}{lcccc}
\hline Material layer & PZT $(\mu \mathrm{m})$ & PMN-PT $(\mu \mathrm{m})$ & Ni chrome $(\mu \mathrm{m})$ & $\mathrm{Au}(\mu \mathrm{m})$ \\
\hline Thickness & $102 \pm 10$ & $102 \pm 10$ & $0.09 \pm 0.03$ & $0.175 \pm 0.06$ \\
Length & $1330 \pm 13$ & $1330 \pm 13$ & $1330 \pm 13$ & $1330 \pm 13$ \\
Width & $1016 \pm 13$ & $1016 \pm 13$ & $1016 \pm 13$ & $1016 \pm 13$
\end{tabular}

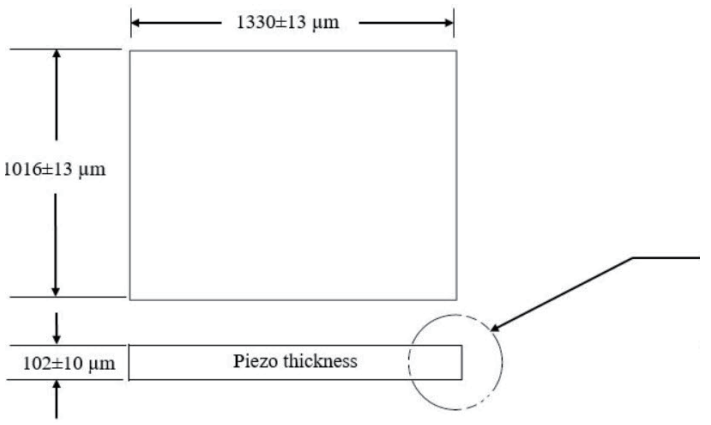

(a)

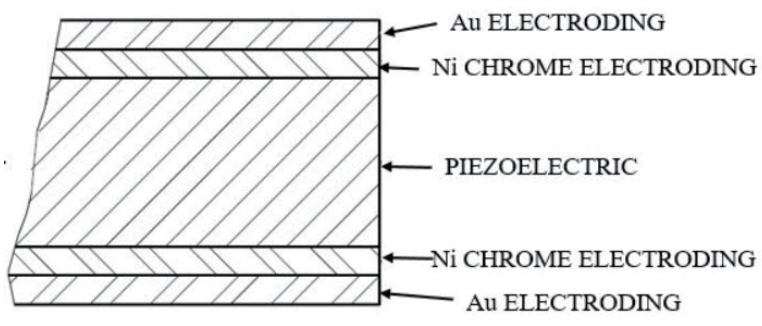

(b)

Fig. 3. (a) Piezoelectric material dimensions and (b) cross-sectional view.

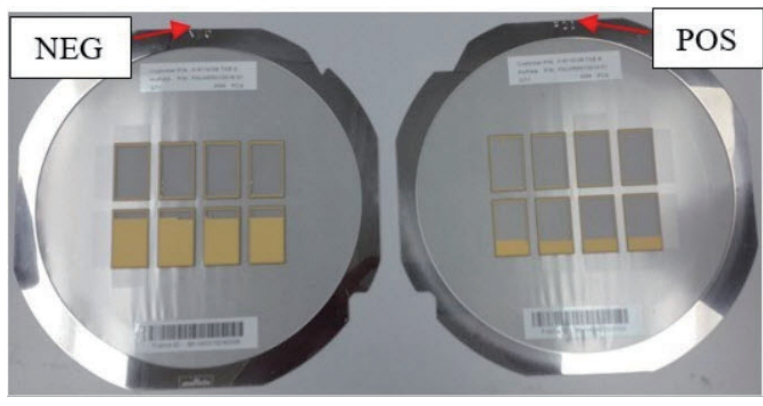

(a)

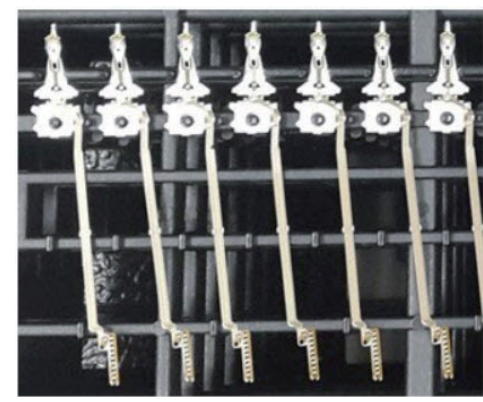

(b)

Fig. 4. (Color online) (a) Plate piezoelectric wafer and (b) raw HGA.

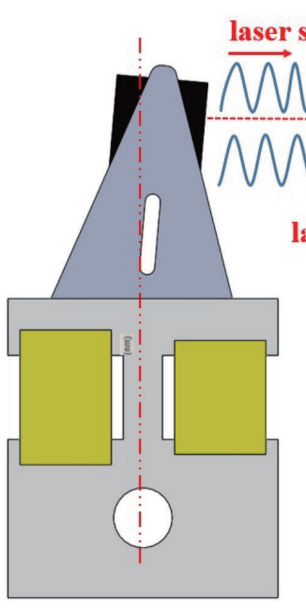

(a)

\section{LDV}

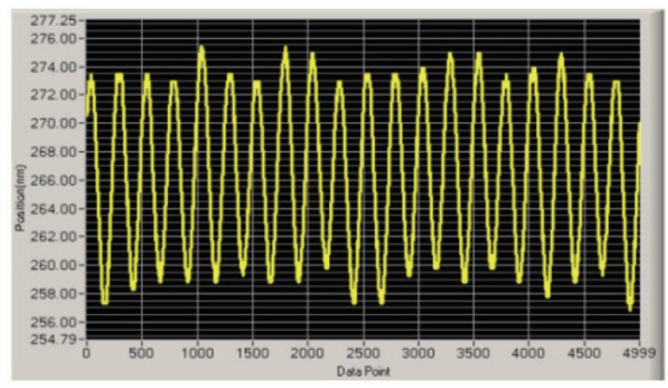

(b)

Fig. 5. (Color online) (a) LDV sensing of slider head and (b) raw data from LDV. 


\section{Piezoelectric Die Assembling Steps}

The second-stage actuator of an HGA consisted of two piezoelectric dies of which the poling direction of the left die was positive and that of the right die was negative. The top surface was connected to the electrical ground with a conductive adhesive, while the bottom surface was connected to the positive pole of the driver circuit also with a conductive adhesive. At the time of the study, a suspension-based actuator was assembled by a pick-and-place machine. As shown in Fig. 6(a), the piezoelectric dies were bonded to the load beams of the HGA. Piezoelectric dies must be placed very carefully and accurately so as to avoid short-circuiting the piezoelectric or creating a non-ohmic electrical contact and to not cause a crack.

The three die assembling steps were as follows.

\subsection{Dropping nonconductive and conductive adhesives on the HGA}

A few drops of nonconductive adhesive, Loctite ablestik 2035SC-1B1, were applied to the load beam of HGA for securing the piezoelectric material to it and preventing short-circuiting, while a drop of conductive adhesive, Loctite ablestik 2030SC, was applied to each circuit jumper shown in Fig. 6(b) to electrically connect the piezoelectric die to the positive pole of the driver circuit.

\subsection{Attaching the piezoelectric die}

The piezoelectric actuator was assembled by a pick-and-place machine that placed piezoelectric dies on the load beam of the HGA that was already applied with adhesives to within stringent placement tolerances, as shown in Figs. 7(a) and 7(b). The piezoelectric dies sat on a nonconductive adhesive layer that acted as an adhesive "dam" between the surface of the piezoelectric material and that of the load beam. The nonconductive adhesive overflow distance was allowed to be up to $0.1 \mathrm{~mm}(0.004$ ") from the top edge of the piezoelectric plate to its body and from the top edge of the piezoelectric plate to the base plate as shown in Fig. 7(a). Similarly, the nonconductive adhesive overflow distance at the bottom edge of the piezoelectric plate was

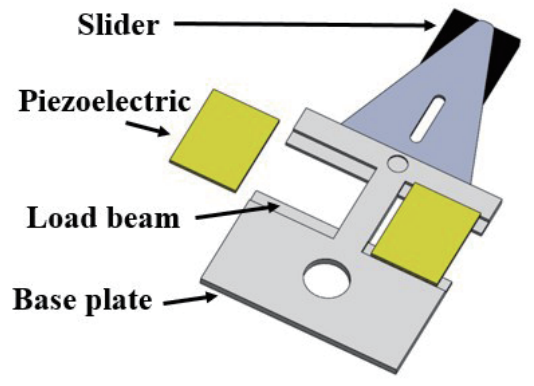

(a)

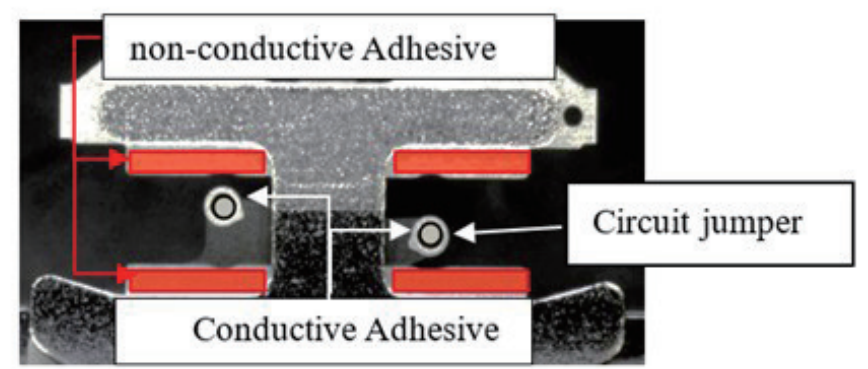

(b)

Fig. 6. (Color online) (a) Schematic of HGA and (b) area for applying adhesives. 


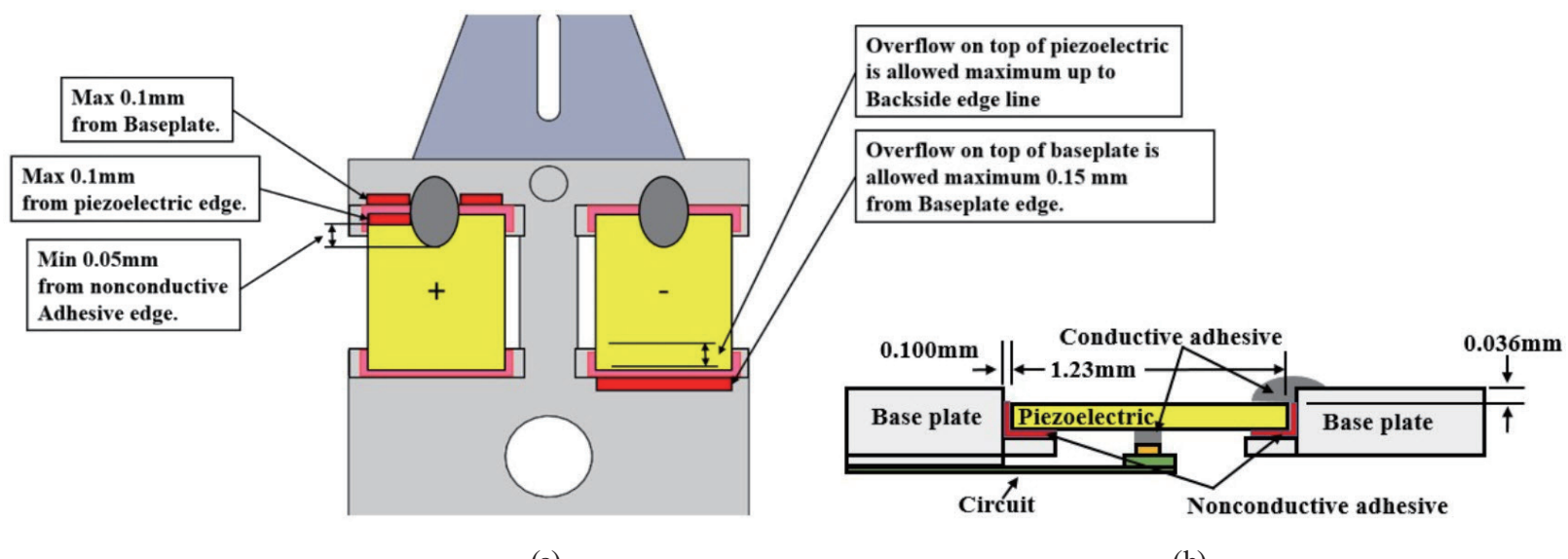

(a)

(b)

Fig. 7. (Color online) (a) Tolerances of adhesive application and (b) cross section of the side view of HGA.

allowed to be $0.15 \mathrm{~mm}(0.006$ "). Figure 7(b) shows the tolerance of the gap between the side of the base plate and that of the piezoelectric plate of $0.100 \mathrm{~mm}$ at both sides and the tolerance of the height of the piezoelectric plate relative to the height of the base plate of no more than 0.036 $\mathrm{mm}$.

\subsection{Electrically connecting the piezoelectric plate to the driver circuit}

Electrical connection points between the HGA and the piezoelectric plate appeared as a raised conductive adhesive "line" that also physically secured the piezoelectric plate to the HGA, as shown in Fig. 8(a). If the conductive adhesive did not make good contact with the plate and HGA, the part would be rejected. A minimum adhesive line length of $0.05 \mathrm{~mm}$ (0.002") was required to ensure good electrical ground connection. Also, the circuit jumpers at the bottom side of the piezoelectric plate needed to be verified to make good electrical contact as shown in Fig. 8(b). The verification was performed by an LCR meter, but the details of the verification procedure are not reported in this paper, but will be reported in our next paper.

\section{Experimental Procedures and Results}

HGA performance concerns its dynamic and static behaviors. The most important parameter of its dynamic behavior is its resonance frequency of a certain vibrational mode. For our purpose, it was sufficient to determine whether the resonance frequency of this developed HGA fell in the manufacturing specification range, because if it did, there would be no need to modify the manufacturing process. The base plate was excited by using a shaker and the lateral motion of the slider was measured by a Polytec OFV-534 LDV and the resonance frequency of each mode of interest was determined and displayed as a graph by the HGA testing system. The most important parameter of the HGA static behavior is its stroke travel range. To measure it, only the piezoelectric plates themselves were excited by applying a sinusoidal voltage at $20 \mathrm{Vp}-\mathrm{p}$ 


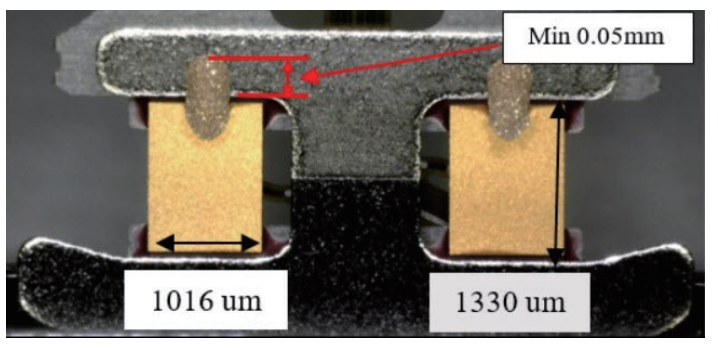

(a)

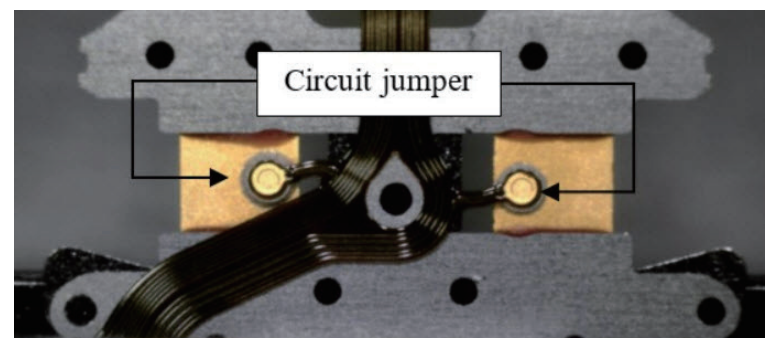

(b)

Fig. 8. (Color online) (a) Specifications of conductive adhesive line on the top surface of the piezoelectric plate and (b) points of contact of the circuit jumpers.

to them and the displacement range of the slider was determined by the same LDV. In the next two sections, the experimental procedures and the measurement results of these parameters are reported in detail.

\subsection{Resonance analysis}

A servo control system controls the position of the slider of the HGA over a track on the magnetic disk. HGA resonant frequencies are very important to the overall stability of the servo control system and the settling and seek performance characteristics of the drive system. They depend on several HGA design features such as length, thickness, mass, rail height, spring rate, and mass distribution.

An HGA has many natural vibrational modes depending on a particular design. Normally, an HGA design would have the following five important mode shapes: 1st bending, 1st torsion, 2nd bending, 2nd torsion, and sway. Resonant mode shapes are considered to be the most critical HGA design parameters that are optimized during the design phase and monitored during the production cycle as items of specifications. The specifications of our design exactly followed those of the manufacturer and are shown in Table 3.

For measuring the resonance frequencies, the HGA testing system used several devices, namely, a shaker assembly, a spindle stand, an LDV, a video camera, a signal analyzer (Matlab/ Labview), and an industrial PC, as shown in Fig. 9.

HGA resonant measurement is an open loop measurement of the transfer function between the input at the baseplate and the output at the R/W head [see Fig. 10(a)]. The settings of the tester were as follows: a sinusoidal frequency range of $1-50 \mathrm{kHz}$, a disk spindle speed of 10000 $\mathrm{rpm}$, and a flying distance of $0.635 \mathrm{~mm}$ between the disk and the slider head ( $\mathrm{Z}$ height). The measurement operation started with the shaker excitation of the baseplate of the HGA, hence off-track motion was generated by the shaker and the displacement, velocity, and acceleration of the slider were determined. The shaker provided a persistent excitation of the desired frequency bands with adequate energy.

Figure 10(b) shows $f(t)$, the baseplate velocity excited by the shaker, and $x(t)$, the slider velocity measured by the LDV. The relationship between the gain (the logarithmic ratio 
Table 3

Specifications of resonance frequency of important modes.

\begin{tabular}{lcrrr}
\hline Resonance mode & Unit & \multicolumn{1}{c}{ Min. } & Target & Max. \\
\hline 1st bending mode & $\mathrm{Hz}$ & 7350 & 8150 & 8950 \\
1st torsion mode & $\mathrm{Hz}$ & 14700 & 15500 & 16300 \\
2nd bending mode & $\mathrm{Hz}$ & 16800 & 16800 & 17600 \\
2nd torsion mode & $\mathrm{Hz}$ & 19000 & 20800 & 21600 \\
Sway mode & $\mathrm{Hz}$ & 21600 & 22800 & 24000 \\
\hline
\end{tabular}

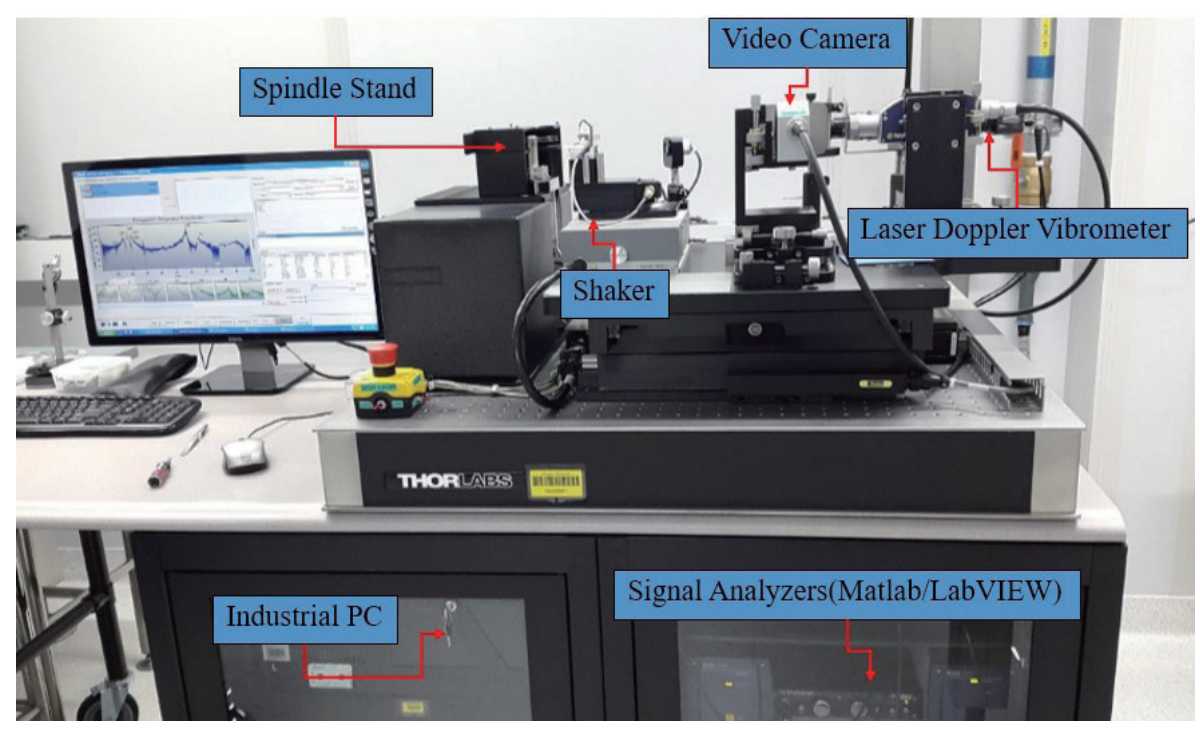

Fig. 9. (Color online) HGA tester overview.

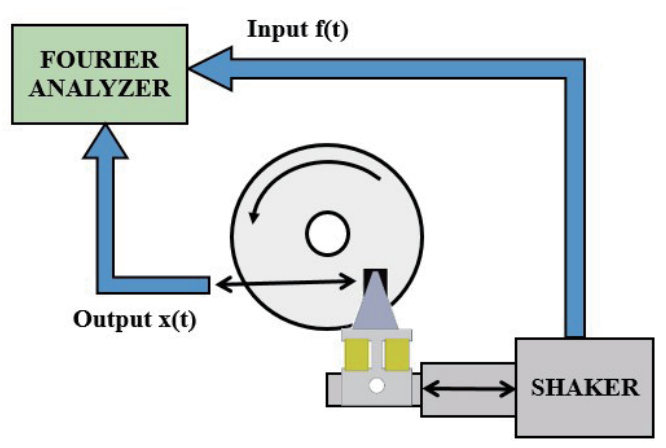

(a)

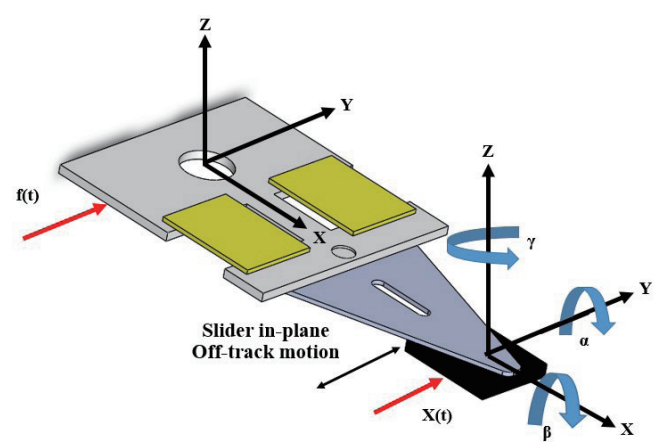

(b)

Fig. 10. (Color online) Transfer function measurement of HGA: (a) process diagram and (b) directions of shaker modes mapping to the slider.

between base plate and slider velocities) and the HGA vibrational frequency is shown in Figs. 11 (for PZT) and 12 (for PMN-PT). If the base plate and slider velocities are expressed in the same unit, then the limit of the gain as the frequency approaches zero is zero. 


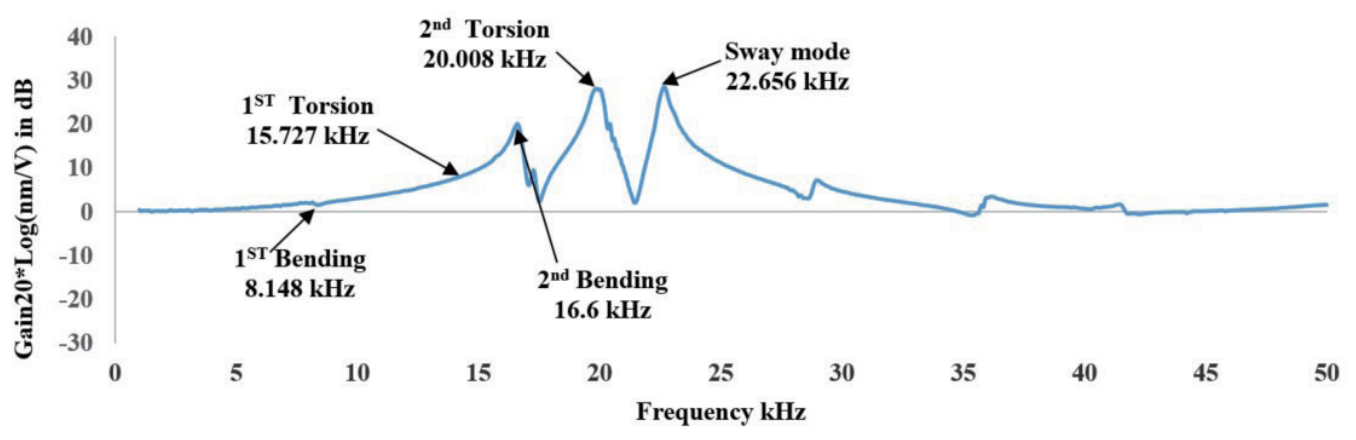

Fig. 11. (Color online) Resonance frequencies of PZT-based HGA.

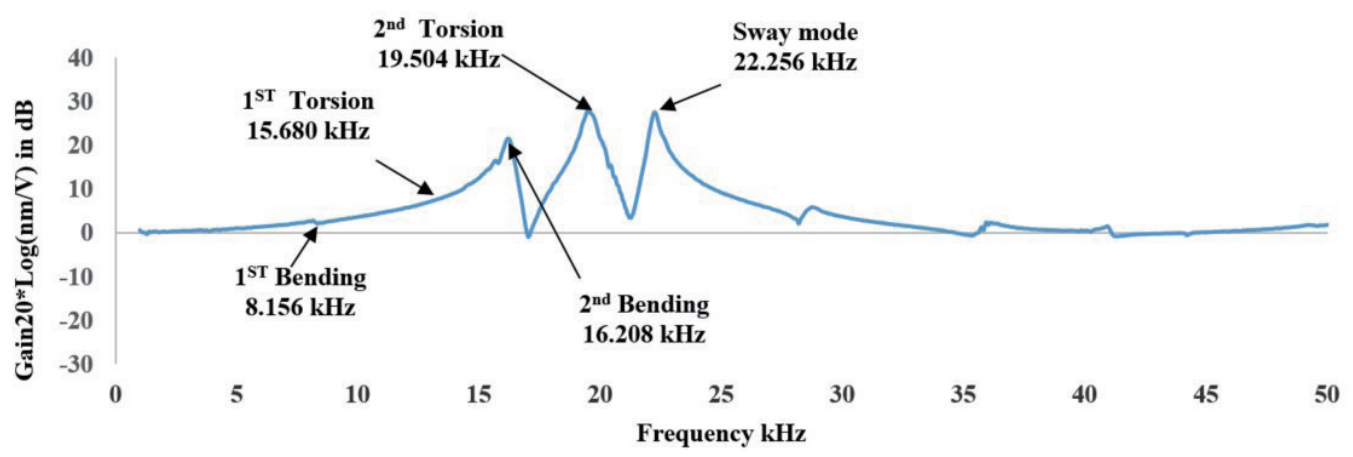

Fig. 12. (Color online) Resonance frequencies of PMN-PT-based HGA.

\subsection{Stroke analysis}

The maximum stroke of a microactuator affects the settling and track-following performance characteristics of a design. In this work, the piezoelectric plates were excited by a sinusoidal voltage at $20 \mathrm{Vp}-\mathrm{p}$ at $800 \mathrm{~Hz}$ for 10 cycles in order to reduce the chance of electrostatic discharge in the HDD as shown in the flow diagram in Fig. 13.

The stroke and hysteresis data were collected by pointing a laser beam to the R/W head that was being tested, and the reflected beam was detected by a laser interferometer system, which outputted the displacement for each input voltage as shown in Fig. 14.

\section{Discussion}

The most important performance characteristics of a microactuator are considered to be those of its dynamic and static behaviors. The performance parameters of its dynamic behavior investigated here were its resonance frequency and slider head gain. Shown in Table 4 are resonant frequencies of five vibrational modes of PZT and PMN-PT microactuators and their corresponding slider head gains. Since the resonance frequencies were not much different, indicating that their mechanical stiffnesses were not very different, modal performance characteristics such as the resonance frequency of the HGA were dominant and it was not 


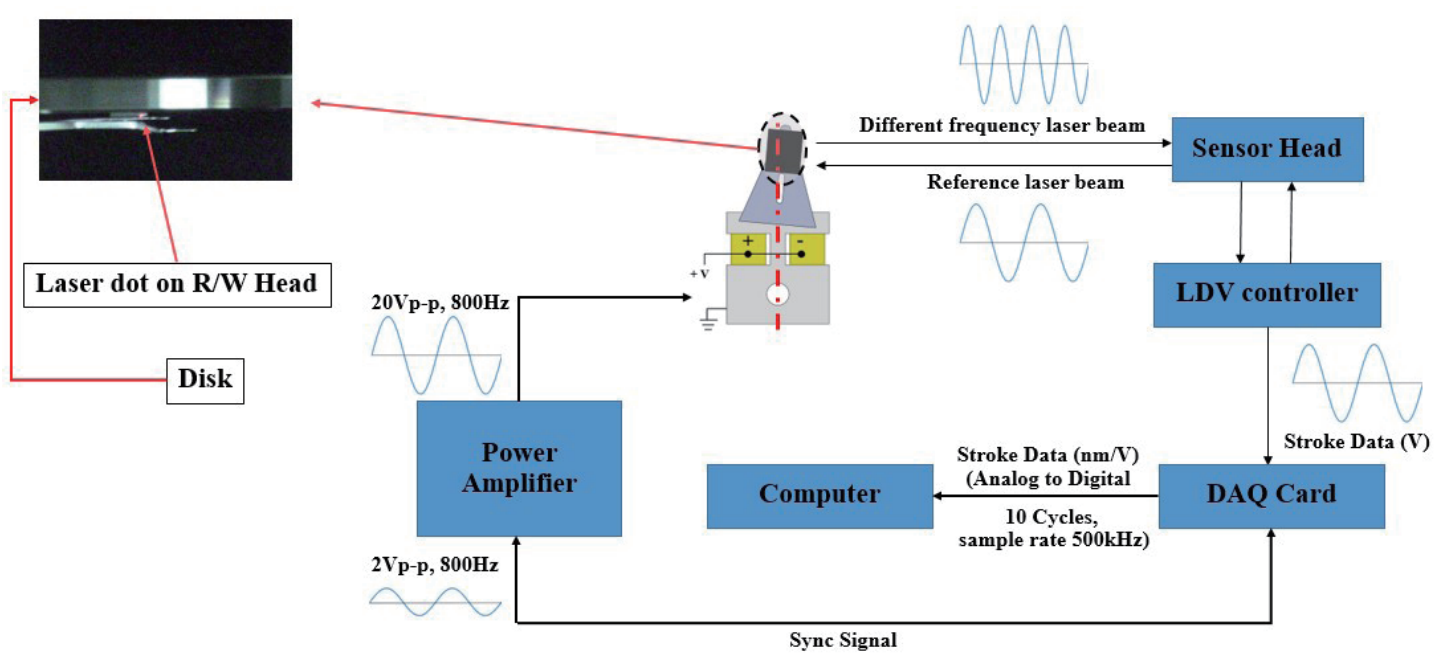

Fig. 13. (Color online) Flow diagram of stroke analysis procedure.

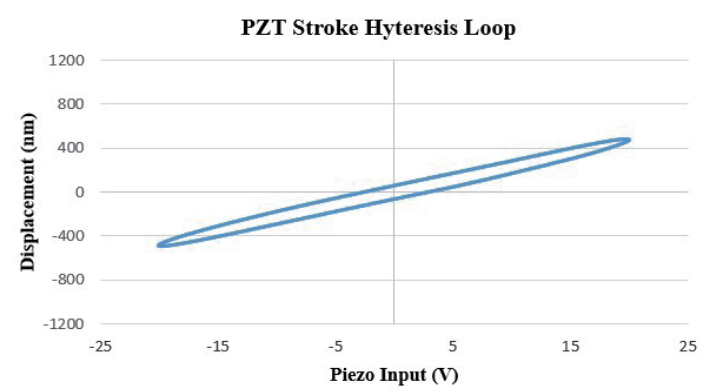

(a)

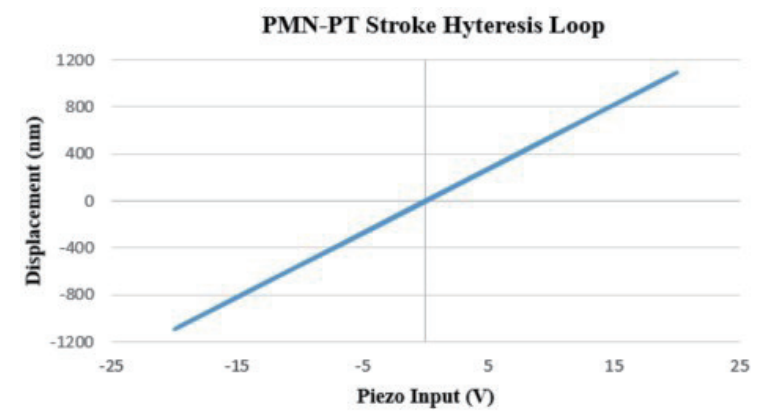

(b)

Fig. 14. (Color online) Measured response of stroke linearity and hysteresis loop.

Table 4

Results of frequencies and gains of PMN-PT and PZT actuators.

\begin{tabular}{lcccc}
\hline Mode & \multicolumn{2}{c}{ Frequency $(\mathrm{kHz})$} & \multicolumn{2}{c}{ Gain $(\mathrm{dB}-\mathrm{nm})$} \\
\cline { 2 - 5 } & \multicolumn{1}{c}{ PZT } & PMN-PT & \multicolumn{1}{c}{ PZT } & PMN-PT \\
\hline 1st bending mode & 8.148 & 8.156 & 1.874 & 2.237 \\
1st torsion mode & 15.727 & 15.680 & 14.194 & 14.610 \\
2nd bending mode & 16.600 & 16.208 & 19.628 & 21.43 \\
2nd torsion mode & 20.008 & 19.504 & 27.825 & 26.573 \\
Sway mode & 22.656 & 22.256 & 27.581 & 26.173 \\
\hline
\end{tabular}

necessary to rewrite the notch filter in the servo code in order to replace the PZT actuator with the PMN-PT actuator in the HDD as well as to change the original HGA design. Moreover, the gain levels of the PMN-PT microactuator were not much different from those of the PZT microactuator, signifying that this new actuator can replace the traditional PZT actuator without any need to redesign the mechanical structure of the HGA. 
Table 5

Results of stroke travel and area of hysteresis loop of stroke travel of the PMN-PT and the PZT microactuators.

\begin{tabular}{|c|c|c|c|c|c|}
\hline \multicolumn{2}{|c|}{ Peak voltage $(\mathrm{V})$} & \multicolumn{2}{|c|}{ Peak stroke $(\mathrm{nm} / \mathrm{V})$} & \multicolumn{2}{|c|}{ Hysteresis area (V-nm) } \\
\hline PZT & PMN-PT & PZT & PMN-PT & PZT & PMN-PT \\
\hline 20.06 & 20.06 & 24.36 & 54.61 & 4191.20 & 419.80 \\
\hline
\end{tabular}

For the static behavior, the performance parameters investigated were stroke travel and the hysteresis loop of stroke travel. In general, a wider range of slider head stroke travel results in desirable lower track seeking time and track-seeking error rate because a single actuation can cover a larger number of data tracks, which were the purposes of the development of this new actuator. In the same vein, a smaller area of the hysteresis loop of travel makes it easier and faster to control the movement of the slider head because of the more linear control characteristic, which was also the other purpose of this development. The results in Table 5 show that the peak stroke travel enabled by the PMN-PT-based actuator was more than two times longer than that provided by the original PZT-based actuator, and the area of the hysteresis loop provided by the new actuator was smaller than that provided by the original PZT actuator. This longer stroke travel afforded by PMN-PT is in full agreement with an investigation by Kim et al., ${ }^{(18)}$ which demonstrated that the tip displacement of a unimorph actuator provided by PMN-PT was 15 times larger than that provided by PZT. From all of the better performance results mentioned above, we concluded that this new PMN-PT microactuator is a step above the original PZT microactuator and can readily replace the PZT microactuator.

\section{Conclusions}

In this research study, PMN-PT was implemented in a DSA of HDD. This was the first time that PMN-PT was investigated in an actual comparative experiment with traditional PZT as a microactuator of an HGA of HDD. Comparisons were made on 2 parameters of their dynamic behavior - resonance frequencies and slider head gains of 5 vibrational modesand 2 parameters of their static behavior-slider head stroke travel and area of the hysteresis loop of stroke travel. The experimental results of all 4 parameters indicate that this new PMNPT actuator performs better than the traditional PZT actuator; in particular, it has a more than 2 times wider stroke travel range than the PZT actuator. Moreover, this new technology can be applied to current manufacturing lines with no need to upgrade the existing assembling machines and can enable the fast and precise positioning of the $\mathrm{R} / \mathrm{W}$ magnetic head of extremely high density HDD for large data storage in the future. Besides demonstrating the superiority of PMN-PT over PZT as a microactuator, the comparative data of dynamic and static behaviors of PMN-PT versus PZT obtained in this study can be put to good use by sensor designers. Moreover, in current research works on cyber-physical systems, PZT has been used as sensors and actuators for many types of systems such as wireless piezoelectric sensors and actuators for structural health monitoring ${ }^{(19)}$ and energy harvesting. ${ }^{(20)}$ The data from our study should point a way for researchers in search of a better-performing piezoelectric material to take interest in PMN-PT as a practical replacement of PZT. 


\section{Acknowledgments}

This work was financially supported by the Thailand Research Fund and Seagate Technology Thailand (No. PHD5810005). The authors would also like to thank Seagate Technology Thailand for providing excellent research facilities for this study.

\section{References}

W. Guo, T. Huang, C. Bi, K. T. Chang, and T. S. Low: IEEE Trans. Magn. 34 (1998) 1907.

Y. Soeno, S. Ichikawa, T. Tsuna, Y. Sato, and I. Sato: IEEE Trans. Magn. 35 (1999) 983.

3 Y. M. Niu, W. Guo, G. X. Guo, H. O. Eng, K. K. Sivadasan, and T. Huang: IEEE Trans. Magn. 36 (2000) 2241.

4 K. Mori, T. Munemoto, H. Otsuki, Y. Yamaguchi, and K. Akagi: IEEE Trans. Magn. 27 (1991) 5298.

5 C. K. Thum, C. Du, J. Zhang, K. P. Tan, B. M. Chen, and E. H. Ong: IEEE Trans. Magn. 44 (2008) 2227.

6 R. B. Evans, J. S. Griesbach, and W. C. Messner: IEEE Trans. Magn. 35 (1999) 977.

7 S. Koganezawa, Y. Uematsu, and T. Yamada: IEEE Trans. Magn. 35 (1999) 988.

8 D. K. Miu: Data Storage Mag. 4 (1995) 33.

9 D. A. Horsley, N. Wongkomet, R. Horowitz, and A. P. Pisano: IEEE Trans. Magn. 35 (1999) 993.

10 P. Panthongsy and D. Isarakorn: Proc. 2016 Int. Conf. Electrical Engineering/Electronics, Computer, Telecommunications and Information Technology (ECTI-CON 2016).

11 B. H. Kim and K. Chun: J. Micromech. Microeng. 11 (2001) 1.

12 L. Chengkuo, I. Toshihiro, and S. Tadatomo: Sens. Actuators, A 72 (1999) 179.

13 S. Katsuhiro and M. Takeshi: Sens. Actuators, A 226 (2015) 760.

14 Y. Li, R. Horowitz, and R. Evans: IEEE Trans. Magn. 39 (2003) 932.

15 C. Huang, Y. Lin, and T. A. Tang: J. Micromech. Microeng. 14 (2004) 530.

16 S. Mei: US Patent 6239953 (2001).

17 P. Wattananukulchai and D. Isarakorn: Proc. 2016 Int. Conf. IEEE Region 10 (TENCON2016).

18 K. C. Kim, Y. S. Kim, H. J. Kim, and S. H. Kim: J. Curr. Appl. Phys. 6 (2006) 1064.

19 A. Abdelgawad and K. Yelamarthi: Proc. 2016 Int. Conf. Circuits and Systems (MWSCAS2016).

20 G. M. Siddesh, G. C. Deka, K. G. Srinivasa, and L. M. Patnaik: Cyber-Physical Systems-A Computational Perspective: Energy Sources (CRC Press, Florida, 2015) p. 192.

\section{About the Authors}

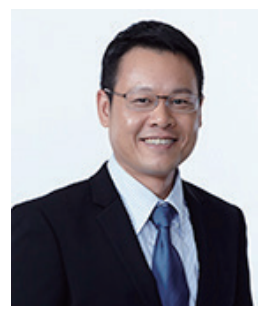

Don Isarakorn is currently an assistant professor of Electrical Engineering in King Mongkut's Institute of Technology Ladkrabang (KMITL), Bangkok, Thailand. He is also the Head of the Multi-Scale Electromechanical Systems Laboratory of the Department of Instrumentation and Control Engineering, where he leads the activities on mechatronic system integration. He received his B.Eng. degree in electronics engineering and his M.Eng. degree in control engineering from KMITL in 2000 and 2003, respectively, and his Ph.D. degree in piezoelectric microelectromechanical systems from the Sensors, Actuators, and Microsystems Laboratory, École Polytechnique Fédérale de Lausanne, Lausanne, Switzerland, in 2011. His main research interests include piezoelectric materials for sensing and actuating applications, energy harvesting systems, robotics, mechatronics, automatic control systems, and biomedical instruments. 


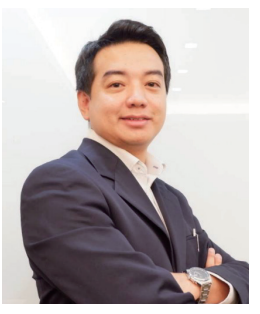

Parinya Wattananukulchai is currently an innovation technology advisor of the innovation center in SME Development Bank and King Mongkut's Institute of Technology Ladkrabang (KMITL), Bangkok, Thailand. He has been working on HSA metrology and as a mechanical engineer in Seagate Technology (Thailand) Ltd., an NPI engineer in Focuz Manufacturing Co., Ltd., a tooling and maintenance engineer in Hitachi Global Storage Technologies Thailand Co., Ltd., and a process engineer in Fabrinet Co., Ltd. He received his M.Eng. degree in mechanical engineering from Chulalongkorn University and B.Eng. degree in mechatronic engineering from KMITL in 2009 and 2005, respectively. Now, his research interests focus on the design, performance, and evaluation of advanced PMN-PT piezoelectrics for dual-stage actuators in hard drive heads, robotics, mechatronics, automatic control systems, and biomedical instruments. 